Chapter 4

\title{
Hypertensive Emergencies
}

\author{
Tomas Janota \\ Additional information is available at the end of the chapter \\ http://dx.doi.org/10.5772/59005
}

\section{Introduction}

The severest hypertensive states pose an immediate threat to life. To be able to manage these situations, physicians need well-defined recommendations; however, as with some other emergencies, only few evidence-based strategies have been developed to date.[1] As randomized, placebo-controlled trials are very difficult to design and conduct, most guidelines and recommendations are based just on experience. The recommendations and opinions in the present chapter draw mainly from national, European, and American guidelines for the treatment of hypertension, and on guidelines of professional societies such as the European Stroke Organisation as well as the experience gained in the author's health-care center.[2-6]

\section{Terminology}

The terminology used for severe hypertensive states is not fully consistent, clear, and systematic. Severe hypertension-associated states can be divided into hypertensive emergencies and hypertensive urgencies under the general title a hypertensive crisis.

Hypertensive emergencies (HE) referring to more severe hypertensive states are defined as an acute, life-threatening states due to a sudden marked rise in blood pressure (BP) resulting in complaints and damage to the structure of some organs. [2-6] High BP leads to the development of acute problems and presentation of organ damage when reaching systolic and diastolic BP usually greater than $220 \mathrm{~mm} \mathrm{Hg}$ and $120 \mathrm{~mm} \mathrm{Hg}$, respectively. However, in individuals so far normotensive, a hypertension may lead to a critical condition such as eclampsia already at a systolic BP of $170 \mathrm{~mm} \mathrm{Hg}$. The severity of the condition is dependent mainly on organ damage, not solely on the level of BP. The immediately threatened vital organs include the central nervous system and the cardiovascular system. Any damage to other organs 
(particularly the kidneys and eyes) is essentially always of primarily vascular etiology; hence, these organs are referred to as target ones. It is reasonable to refer to manifestation of damage since milder damage is often likely to go undetected. Importantly, damage to the central nervous and cardiovascular systems may pose an immediate threat to life. However, even manifestation of damage to another organ is associated with an increased risk of sudden damage to organs performing vital functions.[2-6]

According to the guidelines of the European Society of Hypertension (ESH) and the European Society of Cardiology (ESC), hypertensive emergencies comprise: hypertensive encephalopathy, hypertension with heart failure (including acute so-colled flash pulmonary oedema conditioned by sudden increase in BP), hypertension in acute coronary syndromes, hypertension in aortic dissection, hypertension in intracranial hemorrhage and ischemic stroke, sympathetic crises due to pheochromocytoma or an abuse of so-called recreational drugs (amphetamine, LSD, cocaine, ecstasy/MDMA etc.), perioperative hypertension, eclampsia/ severe pre-eclampsia and acute renal failure in hypertension (Table 1). Some listed HE are not accompanied by organ damage but the instability of the circulatory status and threats to the structure and function of organs belongs them between HE. In the European guidelines, the list of situations classified as HE is preceded by the word "primarily “[4]; hence the item is open for inclusion of other conditions. The current definition also seeks to avoid taking a clear position on the causality of hypertension and organ dysfunction as the degree of causality is frequently unclear.

Hypertensive ugencies should be characterized by complaints yet without manifest organ damage, with BP greater than 180/110 $\mathrm{mmHg}$. These states include accelerated and malignant hypertension, postoperative hypertension, and hypertension in chronic heart failure. In addition to high BP (diastolic BP typically greater than $140 \mathrm{~mm} \mathrm{Hg}$ ), accelerated hypertension is characterized by vascular injury presenting with retinal hemorrhage and exudate formation. The malignant hypertension is more over associated with papilledema and/or renal injury and encephalopathy, manifestations of organ damage characteristic for HE. [2-6] From this perspective, it seems questionable resolution $\mathrm{HE}$ and hypertensive urgencies. The most recent joint ESH a ESC guidelines no longer list the term urgent hypertensive state as it usually does not require specific therapeutic strategies and hospitalization in the intensive care unit (ICU). Management of it is based on stepping up standard therapy with oral medications. Only in cases where it fails or in specific cases not allowing oral administration is parenteral treatment used.[4]

\section{Etiology and prevalence of hypertensive emergencies}

A role in the etiology of emergent hypertensive states is most often played by inadequate treatment of known hypertension and therapy discontinuation. [2,3,7] Pathophysiological mechanisms include a sudden rise in peripheral vascular resistance and systemic vasoconstriction due to activation of the renin-angiontension-aldosterone system, sympathoadrenal system, endothelin, exogenous factors, injury to the central nervous system, bleeding, tumor 
or ischemia, or perioperative stress. Except for conditions associated with renal failure, increased natriuresis and vasoconstriction result in reduced vascular filling. This can be effectively managed by afterload reduction; diuretic use is less effective. Hypertensive encephalopathy develops due to cerebral edema, which forms once the autoregulatory capacity of blood flow through brain arteries has been exceeded. The autoregulatory threshold is typically a mean BP of $140 \mathrm{~mm} \mathrm{Hg}$; however, the value may be higher in protracted severe hypertension. Other factors contributing to the development of edema include both hydrostatic mechanisms and changes in the vascular wall.[8]

The exact prevalence of severe hypertensive states is not known. These states occur more frequently in patients with nephrogenic hypertension and hyperaldosteronism. An emergent hypertensive status develops in about $1 \%$ of hypertensives during their lifetime.[9] An earlier US study reported about $8 \%$ and $16 \%$ of patients presenting at medical part of the emergency department with emergent and urgent hypertensive states, respectively.[10] In the experience of the author of this chapter, about $5 \%$ of patients admitted to the Intensive Cardiac Care Unit are diagnosed to have states somehow related to severe hypertension.

\section{Clinical picture}

The most frequent complaints accompanying HE include chest pain (27\%), dyspnea (22\%), headache $(22 \%)$, epistaxis $(17 \%)$, weakness and psychomotorical agitation $(10 \%)$, whereas other authors list headache $(49 \%)$ and weakness $(22 \%)$ as the most frequently experienced problems.[5,8] Other complaints include palpitations, nausea, vomiting, anxiety and feeling of illness. Organ damage manifests itself most often as cerebral stroke $(24 \%)$, lung edema $(23 \%)$, neurological deficit (21\%), and hypertensive encephalopathy (16\%). Cerebral hemorrhage is diagnosed as little as $4.5 \%$ of cases (Tab. 2).[10] Retinal hemorrhage, exudates, and papilledema can also occur, indicating damage to other organs. These lesions do not subside until after several weeks since BP reduction. Oliguria, hyperazotemia, and proteinuria signal renal injury. Patients complaining of concomitant chest pain or other discomfort or with EKG-documented ST-segment depressions and a mild increase in cardiac troponins in serum can be diagnosed, according to the Universal definition of myocardial infarction, as type 2 myocardial infarction due to an imbalance between oxygen demand and supply.[11]

\section{Examinations}

Given the possibility of coarctation, atheorsclerosis of brachial arteries or, possibly, aortic dissection, BP should be measured on both arms. Measurement of BP in the lower limbs will rule out stenosis of arteries in both arms, a rare occurence. Blood pressure is measured using a properly-sized cuff to match the limb circumference. During subsequent managements in the ICU, BP is commonly measured noninvasively at intervals of 5 minutes to 1 hour depending on the severity of organ damage and BP level and instability. Only in cases of persisting 
instability of BP and its difficult control, or with conditions such as dissecting aneurysm requiring controlled hypotension, is it necessary to insert an arterial line. The cannula should be preferably inserted after a reduction of BP by initial therapy in some compressible brachial artery. An integral part of physical examination is neurological assessment to guide therapy. Basic biochemical tests including plasma concentration of minerals, urea, creatinine, markers of myocardial necrosis, natriuretic peptides, liver tests (to determine elimination of drugs), urinalysis (albuminuria, hematuria, crystaluria, etc.), and blood count should be done immediately. Additional examinations include EKG, chest x-ray, and fundoscopy. Echocardiography is performed in the context of hypertension primarily to assess the presence of myocardial dysfunction, muscle hypertrophy or, possibly, to roule out dissection of the aorta. Patient diagnosed to have focal neurological deficit and encephalopathy require a brain CT. Additional examinations are performed based on the patient's current status.

\section{Management}

Management of HE should be instituted immediately and continued in ICU. Therapy during transportation to the ICU is of course limited espetially by the possibilities of BP measurement as well as by continuous dousing of IV medication.

The goal of treatment is to reduce $\mathrm{BP}$ and thus to prevent target organ damage and threat of life. However, very high BP must not be reduced very quickly. Too rapid and large BP reduction could be as dangerous and harmful as high BP. Blood pressure should be reduced by only $20-25 \%$ of baseline value over the first hours or until reaching levels 150-160/100-110 $\mathrm{mm} \mathrm{Hg}$. Alternatively, it is recommended to decrease mean BP to below 110-115 mm Hg within 30-60 minutes and, possibly, continue depending on the response and, most importantly, on how the patient tolerates the reduction. An abrupt fall in BP may-primarily in elderly patients with coronary and cerebrovascular atherosclerosis-impair organ perfusion, possibly resulting in acute renal failure, ischemic heart and cerebral events, renal artery occlusion and, eventually, acute blindness. However, in many cases, it is impossible to achieve a steady reduction of $\mathrm{BP}$ as recommended, particularly in settings where invasive monitoring is unavailable. A frequent occurrence is a rapid fall of an initially resistant high BP once contributing factors such as fear, uneasines and pain have disappeared. Achievement of the target BP for the chronic treatment is supposed within 1-2 days.[2-6]

The drugs to be used should have a short half-time of action but a rapid onset of action. They should be administered intravenously. When planning to use a combination of such agents, it is reasonable to have several venous access sites allowing for their separate administration. This often requires the insertion of a central venous line. Some agents such as nimodipine should only be administered via a central venous catheter. On the other hand, any inadvertent puncture of an artery in an effort to cannulate a central vein of a patient with poorly controlled high BP carries the risk of major bleeding.[2-6]

Hypertensive urgencies can usually be managed by intensification of their oral therapy or its complementation. Parenteral therapy is not started unless oral therapy has failed or is 
not accepted. Stay at ICU is not necessary. According to US authors, therapy can even be administered on an outpatient basis with intensive patient monitoring over the first days of therapy. [2,10]

\section{Drugs for IV therapy of severe hypertension}

The agents currently available for parenteral therapy include nitrates, urapidil, diuretics, angiotensin-converting blockers, calcium-channel blockers, beta-blockers, alpha-blockers, combined alpha-and beta-blockers, clonidine and fenoldopam.(Tab. 3) [2,3,6]

Nitrates (isosorbide dinitrateand glycerol-trinitrate) can be the drugs of first choice in almost all conditions. They can be combined with most of the other hypertensive classes and are a reasonable option for pre-hospital initiation of therapy, with a rapid onset of effect within 2-5 minutes to subside within 5-10 minutes. Nitrates are applied as continuous infusion at a rate of $0.5-10 \mathrm{mg} / \mathrm{h}$. At higher doses veno-as well as arteriodilation is present. If given at high doses, the antihypertensive effect is usually not incremental, yet the risk of headache is increased. As the patient becomes tolerant to continuous infusion, nitrates should be stopped discontinued after 2 days.[12] Sublingual nitrate administration may result in too rapid fall of BP; the effect is short-term, hence this use is not reasonable.

Sodium nitroprusside is one of the most effective drugs with an almost immediate onset of effect and a short half-life, with the BP reduction resolving within 2-3 minutes. Infusion therapy is started at $0.2 \mu \mathrm{g} / \mathrm{kg} / \mathrm{min}$. The rate of infusion is every 3-5 minutes doubled to achieve the target BP. The speed of the application usually does not exceed $10 \mu \mathrm{g} / \mathrm{kg} / \mathrm{min}$. The mechanism of its action is vasodilation; however, unlike nitrates, it exerts a more marked effect on arterioles. As a major drawback of sodium nitroprusside is its photosensitivity. It should be infused via dark-color sets, with the infusion bottle covered with a light-tight material. Infusion at a higher rate for 2-3 days, particularly in patients with renal failure, is associated with the risk of intoxication with thiocyanate, a sodium nitroprusside metabolite, manifesting itself with confusion, nausea, and acidosis. Thiocyanate intoxication can be prevented by monitoring its levels. Concentration greater than $10 \mathrm{mg} / 100 \mathrm{ml}$ is toxic. Alternatively, toxicity can be reduced by thiosulfate infusion, continuous infusion of $10 \%$ sodium thiosulfate in a volume ratio of 10:1 (nitroprusside : thiosulfate).[13]

Urapidil hydrochloride is an antagonist of peripheral alpha1-postsynaptic receptors; animal studies have shown it is also an antagonist of central 5-hydroxytryptamine-1A receptors. It has been shown to decrease particularly peripheral vascular resistance withe the resultant decreas both preload and afterload. It has been associated with marked selective renal and pulmonary vasodilation. Unlike other vasodilators, it does not induce appreciable tachycardia, likely due to central modulation of cardiovascular centers via 5-hydroxytryptamin receptores. Its other effects include mild $\beta 1$-blockade. Urapidil decreases systolic and diastolic BP in a balanced manner. The onset of its effect is virtually immediate, peaking within 2-5 minutes. Its antihypertensive effect subsides completely within 4 hours at most. Regarding its use in stroke, it should be noted that, unlike other antihypertensives, urapidil does not exacerbate 
cerebral edema and intracranial pressure. Urapidil is extensively metabolized (50-70\%) in the liver, with $15-50 \%$ of the drug excreted through the kidneys. Side effects are rather rare and mild. The most frequent ones include tachycardia, sweating, chest discomfort, dyspnea, and weakness. If given at higher doses, urapidil has a sedative action. Literary data suggest that its antihypertensive effect is similar to that of enalaprilat, nitroprusside, nifedipine, and dihydralazine. Resistance to urapidil occurs more rarely with with enalaprilate and nifedipine. As a standard policy, therapy with urapidil is initiated with a bolus followed immediately by continuous infusion. It is recommended to give a $10-50 \mathrm{mg}$ bolus over 20 seconds depending on the BP level and clinical status. If there is a sufficient decrease in BP within 5 minutes of bolus administration, continuous therapy can be initiated. In the event of an inadequate effect of the first bolus, especially when rapid BP control is needed, additional boluses should be administered with patient status to be re-assessed at a 5-minute interval. While repeat bolus administration until achieving target BP with subsequent maintenance therapy provide for the quickest BP normalization, the total dose of initial boluses should preferably not exceed $50 \mathrm{mg}$ because of the risk of a sudden severe fall of BP. A dose of $100 \mathrm{mg}$ is only an alternative for very severe resistant hypertension with signs of organ damage. By contrast, in patients raising concern of a major fall in BP and a less emergent status, therapy can be initiated directly with continuous i.v. infusion at a rate of $120 \mathrm{mg} / \mathrm{h}$ without initial bolus administration with a subsequent decrease in the infusion rate once the BP has decreased. It is recommended to continue with maintenance therapy at a rate of $2-10 \mathrm{mg} / \mathrm{h}$ depending on the patient's status and their BP level. The maximum recommended rate for long-term administration is 30 $\mathrm{mg} / \mathrm{h}$. The maximum recommended therapy duration 7 days based primarily on toxicology studies not exceeding the above period of time. Longer administration requires closer monitoring of the patient's status. In the presence of liver or renal failure, it is also recommended only to perform closer hemodynamic monitoring without dose reduction. Given the risk of severe hypotension, combinations of urapidil with another alpha-blocker are contraindicated. Overdose with urapidil or development of side effects can usually be simply managed by dose reduction or therapy discontinuation. In persistent moderate hypotension, the procedure of choice is, of course, volume expansion or, possibly, IV catecholamine administration.

In the case of perioperative hypertension, the recommendation is to initiate therapy with a 25 mg bolus; if effective within 2 minutes of the initial bolus, the patient can be switched to maintenance therapy. The recommended dose of urapidil for maintenance perioperative therapy is $60-180 \mathrm{mg} /$ hour. No data regarding gestational hypertension and urapidil excretion to breast milk are currently available.[14-19]

As urapidil has not yet been approved by the US Food and Drug Administration (FDA), it is simply not mentioned in a number of texts published in the USA.

Clonidine is a centrally-acting drug. It acts primarily via alpha2-receptors to produce protracted vasodilation. Its plasma half-life is in the range of 12 hours. Clonidine could be administered IV as a very slow $150 \mu \mathrm{g}$ bolus (over 10-15 minutes) or infusion at rate 1-2 $\mu \mathrm{g} / \mathrm{kg} / \mathrm{min}$. Maximal daily dose is $750 \mu \mathrm{g}$. At present, its use is reserved for combinations in resistant hypertension and situations requiring sedative effect. Because of the risk of 
potential respiratory center inhibition, it can be used with advantage in patients with mechanical ventilation.[20,21]

Fenoldopam mesylate is a selective D1 dopamin receptor agonist with a half-time of elimination as short as 5 minutes. It causes renal, mesenteric, and coronary arteriodilation while also increasing sodium excretion. Hypotensive efficacy is similar to that of sodium nitroprusside. As a side effect, it produces a tendency to developing tachycardia. However, its co-administration with betablockers may be extremely harmful. Usual initial dosage is $0.1-0.3$ $\mu \mathrm{g} / \mathrm{kg} / \mathrm{min}$. To achieve desired therapeutic effect, may titrate dosage upward or downward in increments of $0.05-0.1 \mu \mathrm{g} / \mathrm{kg} / \mathrm{min}$.[22,23]

Furosemide is a diuretic suitable for emergent situations. Needless to say, its use is appropriate in heart failure and conditions associated with edema formation. As a drug of first choice it is little effective. Ahigh BP induces natriuresis, which, together with frequently concomitant nausea/vomiting, results in fluid depletion and need for hydration rather than diuretic use. Not infrequently is BP reduction seen following saline administration.

Enalaprilate is the only angiotensin-converting enzyme available in injectable form. Its major advantage is its favorable effect on cerebral vascular autoregulation. When administered i.v., its onset of action occurs within 15 minutes, with its peak effect expected after 1-4 hours. Regrettably, the strength and duration of its action are variable lasting as it may 6 but, also 24 hours. Enaprilate is excreted via the kidney. Its dose should be adjusted in patients with renal insufficiency. Contraindications to enaprilate use are identical with those applicable to other angiotensin-converting enzyme inhibitors. As a result, enaprilate is used mostly as a drug of second or third choice in resistant hypertension on the assumption it will not induce undesirable hypotension. The risk of hypotension is particularly high in patients experiencing dehydration. Enalapril is administered as a 0.625 to $1.25 \mathrm{mg}$ bolus, most often at a 6-hour interval depending on the effect. The body of experience with continuous administration is fairly limited.[24]

In outpatient practice, and as a first-aid measure, crushing of a tablet of the short-acting angiotensin-converting enzyme inhibitor captopril at a dose of 25-50 mg may occasionally be helpful. However, in really serious situations with uncertainty regarding future course including the state of consciousness, oral captopril should not be administered.

Calcium-channel blockers have proved effective, particularly in the management and prevention of vasopasms in subarachnoidal hemorrhage. By contrast, concerns have been voiced about their use in ischemic stroke where they may exacerbate collateral cerebral edema. Favorable experience has been obtained with the dihydropyridine-type drugs nicardipine and nimodipine. A drawback of nimodipine is has to be infused via a central venous catheter. Usual infusion rate is $0.5-2.0 \mathrm{mg} / \mathrm{h}$. It is used preferably only in cases of subarachnoidal hemorrhage indicated for neurosurgery. In the event of surgical intervention during treatment, administration of nimodipine should be continued for at least five days. [25] Currently the most promising agent is the novel ultrashort-acting agent clevidipine inducing selective arteriolar dilation including the coronary bed, with onset of action occurring within 2-4 minutes and lasting 5-15 minutes. Dose range between 2 and $16 \mathrm{mg} / \mathrm{h}$. Like nitroprusside or 
calcium-channel blockers clevidipine produce a marked decrease in BP with a mild rise in heart rate and cardiac output. Clevidipine provided in the study ECLIPSE better blood pressure control compared to nitroglycerine and nitroprusside.[26-28] Less experience in emergent hypertensive stats is available with continuous administration of verapamil slowing down heart rate and exerting a somewhat negative inotropic effect. Its use has been suggested in hypertensive states associated with tachycardia at total dose up to $100 \mathrm{mg} /$ day.[29]

Betablockers are the drugs of choice in hypertension and tachycardia. They are particularly advantageous in combination with urapidil or nitroprusside. Betablockers are one of the few classes of drugs appropriate for use in patients with severe hypertension and ischemic stroke. They are traditionally used in the treatment of dissecting aneurysms as they are believed to reduce pulse pressure thereby decreasing aortic wall stress; however, their effect on Dp/Dt has been recently challenged. Betablockers are recommended for use in intraoperative hypertension. Particularly esmolol, highly beta1-selective and ultra-short acting drug with peak effect after administration of $500 \mathrm{mg} / \mathrm{kg}$ bolus followed by continuous i.v. administration reached during 5 minutes and resolving within 10-30 minutes is recommended in HE. The dose is up-titrated at a 5-minute interval with or without boluses. Continuous dose range between 25 and $300 \mu \mathrm{g} / \mathrm{kg} / \mathrm{min}$. [30] Metoprolol in continuous infusion at a rate $1-5 \mathrm{mg} / \mathrm{h}$ could be also effective but with longer hilf-time and lower selectivity compare to esmolol. Boluses of $2.5-5 \mathrm{mg}$ administered one to three times as needed to achieve goal are also frequently used.[31]

Labetalol is both a selective alpha1-blocker and predominantly a non-selective beta-blocker. Treatment is initiated depending on its effect with 5-160 mg boluses administered repeatedly at 2-10 minutes to a maximum dose of $300 \mathrm{mg}$. Labetalol can also be administered continuously at a rate $2 \mathrm{mg} / \mathrm{minutes}$ up to a total dose of $300 \mathrm{mg} / 24$ hours. The effect sets in within 5-10 minutes and resolves within 2-6 hours. A traditional indication-apparently based, in particular, on the absence of adverse experience-is management of pre-eclampsia/eclampsia. It is also the drug of choice in other situations, except those contraindicated to beta-blocker use.[32] In the USA, labetalol is the drug most often used in the treatment of HE.

Alpha-blockerphentolamine is indicated espetially for the control of hypertensive episodes that may occur in a patient with pheochromocytoma as a result of stress or manipulation during preoperative preparation and surgical excision. Boluse dose of $5 \mathrm{mg}$ should be applied fast with BP measurements in 1 minute's intervals during first minutes after administration. Repeated administration could be necessary during surgery.[33]

\section{Management in specific situations}

The choice of individual classes of drugs depends on the underlying disease resulting in HE or it is associated with. To date, only few randomized studies have been conducted evaluating treatment of HE and almost no studies designed for head-to-head comparison of various drugs.[1] 
Ischemic stroke usually results in an increase in BP for 1-4 days. Drug therapy of this type of hypertension is associated with the risk of impairing vascular autoregulation with a subsequent decrease in blood flow in the vicinity of the ischemic focus.[35,36] Besides, patients are also increasingly susceptible to an abrupt fall in BP. The benefit of BP reduction in the acute stroke has not been documented except for situations with a diastolic BP $>130 \mathrm{~mm} \mathrm{Hg}$ conclusively associated with progressive damage to brain structures. It is recommended not to decrease BP until it levels rise above $200-210 / 120 \mathrm{mmHg}$. Within the first hours after hospitalisation, BP should be decreased by $10 \%$ of the baseline value. The target BP is below 180/105 mmHg. As BP increases, the risk of secondary bleeding is also likely to increase, apparently not a rare occurrence even without marked hypertension. In patients indicated for thrombolytic therapy it is recommended to reduce BP below 180/110 $\mathrm{mm} \mathrm{Hg.[35,36]} \mathrm{Substan-}$ tial reduction in BP may be indicated only by the simultaneous occurrence of diseases such as heart failure, acute coronary syndrome or aortic dissection. Fast but short acting i.v. drugs are preferred for the accurate titration of the effect. Drugs with side effect of steal phenomenon, worsening of brain oedema due to brain vasodilator effect are to be excluded. The treatment is recommended especially with medication such as labetalol and betablockers. A good experience is with fast and realtively shortly acting urapidil inspite of the risks of the central sedative effect. Enalaprilat may have a beneficial effect on cerebral vascular autoregulation but it is associated with the risk of inducing undesirable protracted hypotension.

In hemorrhagic stroke, the recommendation is to reduce BP already at levels $160 / 110 \mathrm{~mm} \mathrm{Hg}$, with target BP being 140-160/90 mm Hg.[1] No additional BP correction is necessary once bleeding has been controlled. In the presence of vasospasms, the target BP level is 180-220 mm $\mathrm{Hg}$ once bleeding has been controlled. The drugs used in hemorrhagic stroke are the same as those indicted for ischemic stroke Moreover dihydropyridine calcium-channel blockers can also be used for continuous i.v. therapy, particularly in subarachnoidal hemorrage. Even in the absence of elevated BP, calcium-channel blockers can prevent the development of vasospams, a frequent occurrence in subarachnoidal hemorrhage.[37]

In hypertensive encephalopathy, therapy can be initiated with a nitrate. Other recommended agents include parenteral labetalol, esmolol, and enalaprilate. When administering higher doses of urapidil, one should be aware of its sedative effect carrying the risk of impaired consciousness. High BP reduction results in a prompt resolution of symptoms such as confusion, sleepiness to comatose states, seizures or focal and sensory disorders. A typical up to diagnostic feature of hypertensive encephalopathy is the disappearance of impaired consciousness after diastolic BP reduction below $100-110 \mathrm{~mm} \mathrm{Hg}$. However, it is always critical to exclude another organic cause of symptoms attributed to hypertensive encephalopathy.[2,6]

In congestive heart failure, treatment is initiated with nitrates and diuretics. The drug of choice is enalaprilate or, alternatively, urapidil decreasing, in a desirable manner, simultaneously preload and afterload. In this context, fenoldopam seems to be a most promising drug. An important part of therapy is sedation. In heart failure, BP should be reduced more aggressively to levels below 140/90 $\mathrm{mm} \mathrm{Hg}$.

Acute coronary syndromes in hypertension are managed with parenteral nitrates combined with betablockers or, possibly, with urapidil. A critical consideration in these conditions are 
sedation and pain relief. This therapy should precede more aggressive antihypertensive therapy as a high BP is most often due to pain and anxiety. On the other hand, BP normalization may contribute to reduction of myocardial load and pain relief.

Acute aortic dissection requires reduction of systolic BP to $100 \mathrm{~mm} \mathrm{Hg}$ within 20 minutes, most often using a combination of a nitrate, nitroprusside or urapidil with a betablocker. Even when dissection is only suspected, systolic BP should be lowered to $120 \mathrm{~mm} \mathrm{Hg}$ within 20 minutes. [2,6]

In acute renal failure with severe hypertension, the most effective drugs include urapidil, nitropruside, clonidine, and fenoldopam. $[2,6,38]$ In the presence of hyperhydratio, extracorporeal elimination can be indicated in addition to high-dose furosemide. Aggressive therapy should be initiated particularly in the presence of a sudden rise of azotemia and so-called shadows of damaged red blood cells in the urine and dysmorphous red blood cells in urinalysis in phase contrast imaging. A steady BP reduction is especially important in renal injury.

In pheochromocytome with severe hypertension, an advantageous approach is to initiate therapy with an isosorbide dinitrate-type nitrate. In more severe states, treatment is based on a combination of urapidil with a betablocker. Given its predominantly betablocking action, extra caution should be exercised when using labetalol. The alphablocker phentolamine is employed primarily in surgical removal of pheochromocytoma. [2,6]

Treatment of hypertension induced by amphetamine or cocaine abuse is guided by principles similar to those with pheochromocyte. Excessive release of catecholamines has also been reported. [2,6]

Hypertension induced by other exogenous substances is likely to be associated with vasoconstriction and tachycardia, which is why appropriate therapy includes vasodilatators and drugs with bradycardiac activity. [2,6]

In eclampsia/severe pre-eclampsia, treatment should be started already at BP > 170/110 mm $\mathrm{Hg}$. Labetalol is the traditionally recommended drug. As with a variety of other conditions, treatment can be initiated with a nitrate switching the patient to urapidil with a betablocker in resistant hypertension. Nitroprusside can only be administrated over a short period time. While the effect of verapamil can also be tested but the drug should not be administered with $\mathrm{MgSO} 4$ commonly given in eclampsia because of the risk of aggregation.[2,6]

In intra-operative hypertension, the preferred drug is the ultrashort-acting betablocker esmolol. The drugs of choice include nitrates, urapidil, and dihydropyridine-type calciumchannel blockers. $[2,6]$

A switchover from parenteral to oral therapy should be undertaken once the patient has stabilized to make hospitalization as short as possible. It is reasonable to test the efficacy of the chosen medication for at least 1-3 days in the hospital setting. After discharge, the patient should be followed-up at intervals ranging from one week to several weeks. 


\section{Prognosis}

Uncontrolled malignant hypertension results in $90 \%$ one-year mortality due to heart failure, stroke, or renal failure. There are virtually no data from randomized controlled studies showing how treatment with individual drugs affects mortality and morbidity, and whether any drug is superior to another one. Introduction of novel ultrashort-acting and well tolerated drug are expected to provide the greatest benefit in the near future.

Hypertensive encephalopathy

Hypertension with heart failure

Hypertension in acute coronary syndromes

Hypertension in aortic dissection

Hypertension in intracranial hemorrhage

Hypertension in ischemic stroke

Sympathetic crises due to pheochromocytoma

Sympathetic crises after abuse of so-called recreational drugs (amphetamine, LSD, cocaine, ecstasy/MDMA)

Perioperave hypertension

Eclampsia and severe pre-eclampsia

Acute renal failure in hypertension

Table 1. Hypertensive emergencies

\begin{tabular}{lc}
\hline Complaints & $\%$ \\
\hline Chest pain & 27 \\
Dyspnea & 22 \\
Headache & $22-40$ \\
Nosebleed & 17 \\
Weakness and psychomotor agitation & $10-22$ \\
\hline
\end{tabular}

\section{Organ damage}

Increase in troponin levels above the diagnostic cut-off for AMI (Type-2 AMI)

Stroke

Pulmonary edema 


\begin{tabular}{lc}
\hline Complaints & $\%$ \\
\hline Hypertensive encephalopathy & 16 \\
Cerebral hemorrhage & 4.5 \\
\hline
\end{tabular}

Table 2. Most common manifestations of emergent and urgent hypertensive states

Nitrates (isosorbid-dinitrate, glycerol-trinitrate)

Mechanism of action: venodilatation, at higher doses arteriodilation

Onset of action: maximal effect within 2-5 minutes

Duration of action: 5-10 minutes

Specific indications: medication of choice in almost all situations, mostly first choice medication, advantageous especially for heart failure and acute coronary syndromes

Specific contraindications: ischemic stroke

\section{Sodium nitroprusside}

Mechanism of action: arterio- as well as venodilation

Onset of action: almost immediate, maximal effect within 2-3 minutes

Duration of action: minutes, circulatory half-life about 2 minutes

Specific indications: particularly resistant hypertension, controlled hypotension

Specific contraindications: liver failure, severe cardiovascular stenotic defects, ischemic stroke

\section{Urapidil hydrochloride}

Mechanism of action: vasodilatation (antagonist of peripheral alpha1-postsynaptic receptors, antagonist of central 5hydroxytryptamine-1A receptors), mild beta1-blockade

Onset of action: almost immediate, maximal effect within 2-5 minutes

Duration of action: up to 4 hours

Specific indications: sever and resistant hypertension in almost all situations, controlled hypotension

Specific contraindications: no data regarding lactation

\section{Clonidine}

Mechanism of action: vasodilatation (centrally acting alpha2-agonist), sedative, analgesic and opioid properties

Onset of action: almost immediate

Duration of action: plasma half-life is ranging between 10 and 20 hours

Specific indications: for combinations in resistant hypertension and situations requiring sedative effect Specific contraindications: severe brady-arrhythmias resulting from either sick sinus syndrome or AV block of 2nd or 3rd degree 


\section{Fenoldopam mesylate}

Mechanism of action: selective renal dopamine D1 receptor agonist; renal, mesenteric and coronary arteriodilation, increase of sodium excretion

Onset of action: rapid, most of the antihypertensive effect attained in 15 minutes

Duration of action: quickly reversible, half-life 5-10 minutes

Specific indications: particularly useful in patients with severe hypertension associated with end-organ renal damage or volume overload

Specific contraindications: administration with beta-blockers

\section{Enalaprilate}

Mechanisms of action: angiotensin-converting enzyme, normalization of cerebral vascular autoregulation

Onset of action: within 15 minutes, peak effect after 1-4 hours

Duration of action: 6 - 24 hours

Specific indications: drug of two to third choice in resistant hypertension

Specific contraindication: pregnancy, common contraindications to angiotensin-converting enzyme inhibitors

\section{Nimodipine}

Mechanisms of action: calcium-channel blocker with preferential activity on cerebral vessels; increase of cerebral perfusion, particularly in poorly perfused areas, by arterial dilatation

Onset of action: almost immediate

Duration of action: the half-life is 1.1 - 1.7 hours, the terminal half-life is 5-10 hours

Specific indications: preferably aneurysmal subarachnoidal hemorrhage

Specific contraindication: lack of a central venous catheter

\section{Clevidipin}

Mechanisms of action: calcium-channel blocker; selective arteriolodilatation including coronary arteries

Onset of action: within 2-4 min.

Duration of action: $5-15 \mathrm{~min}$.

Specific indications: rapid reduction of BP in the perioperative setting

Specific contraindication: must not be used in patients with defective lipid metabolism accompanied by hyperlipidemia.

\section{Verapamil}

Mechanisms of action: calcium-channel blocker; decrease in peripheral vascular resistance, without an increase in heart rate as a reflex response

Onset of action: within $5 \mathrm{~min}$.

Duration of action: half-life between 3.5 and 7.4 hours

Specific indications: hypertension with tachycardia and contraindications to beta-blockers 
Specific contraindication: common to calcium-channel blockers

\section{Esmolol}

Mechanisms of action: beta1-selective blocker

Onset of action: maximal effect of the selected dose within $5 \mathrm{~min}$. after bolus

Duration of action: 10-30 min., half-life $9 \mathrm{~min}$.

Specific indications: perioperative hypertension, aortic dissection, ischemic stroke, acute coronary syndrome

Specific contraindication: pregnancy, common contraindications to beta-blockers

\section{Metoprolol}

Mechanisms of action: selective beta-blocker

Onset of action: within $5 \mathrm{~min}$.

Duration of action: to 4 hours, elimination half-life 1-9 hours, average 3.5 hours

Specific indications: aortic dissection, acute coronary syndrome

Specific contraindication: pregnancy, common contraindications to beta-blockers

\section{Labetalol}

Mechanisms of action: non-selective beta-blocker, weak alpha-blocker

Onset of action: within 5-10 min.

Duration of action: 2-6 hours

Specific indications: eclampsia, any severe hypertension

Specific contraindication: common contraindications to beta-blockers

\section{Phentolamine}

Mechanisms of action: non-selective alpha-blocker

Onset of action: minutes

Duration of action: 2-4 hours

Specific indications: during surgical manipulation with phaeochromocytoma

Specific contraindication: common contraindications to alpha-blockers

Table 3. Medication for the management of hypertensive emergencies

\section{Author details}

\section{Tomas Janota*}

Address all correspondence to: tomas.janota@vfn.cz

Cardio ICU, 3rd Department of Internal Medicine, Všeobecná fakultní nemocnice(University Hospital), Prague, Czech Republic 


\section{References}

[1] Perez MI, Musini VM. Pharmacological interventions for hypertensive emergencies: a Cochrane systematic review. J Hum Hypertens 2008; 22: 596-607

[2] Elliott WJ. Clinical features in the management of selected hypertensive emergencies. Prog Cardiovasc Dis. 48: 2006; 316-325

[3] Hypertensive emergency and urgency. Herz 2004 ;29: 354

[4] The Task Force for the Management of Arterial Hypertension of the European Society of Hypertension and of the European Society of Cardiology. Guidelines Committee. 2007 Guidelines for the Management of Arterial Hypertension. J Hypertens 2007; 25: 1105-1187

[5] Joint National Committee on Prevention, Detection, Evaluation, and Treatment of High Blood Pressure. National Heart, Lung, and Blood Institute; National High Blood Pressure Education Program Coordinating Committee: Seventh Report of the Joint National Committee on Prevention, Detection, Evaluation, and the Treatment of High Blood Pressure. Hypertension. 42: 2003; 1206-1252

[6] Janota T. Akutní hypertenzní stavy a jejich terapie Cor Vasa 2011;53,8-9

[7] Bender SR, Fong MW, Heitz S, Bisognano JD. Characteristics and management of patients presenting to the emergency department with hypertensive urgency. J Clin Hypertens. 8: 2006; 12-18

[8] Gardner CJ, Lee K. Hypoperfusion syndrome: Insight into the Pathophysiology and Treatment of Hypertensive Encephalopathy. CNS Spectr 2007; 12: 35-42

[9] Slama M, Modeliar SS. Hypertension in the intensive care unit. Curr Opin Kardiol 2006; 21: 279-287

[10] Zampaglione B, Pascale C, Marchisio M, Cavallo-Perin P. Hypertensive urgencies and emergencies: Prevalence and clinical presentation. Hypertension. 27: 1996; 144-147

[11] K. Thygesen, J.S. Albert, H.D. White, et al.: The Writing Group on behalf of the Joint ESC/ACCF/AHA/WHF Task Force for the Universal Definition of Myocardial Infarction. Third universal definition of myocardial infarction European Heart Journal 33 (2012) 2551-2567.

[12] Isoket.spc.https://www.medicines.org.uk/emc/medicine/1887

[13] Nitroprusside.spc.http://www.medicines.org.uk/emc/medicine/651

[14] Schoetensack W, Bruckschen EG, Zech K. Urapidil. New Drugs Annual: Cardiovascular Drugs. 1983, 19 
[15] Ramage AG. Influence of 5-HT1A receptor agonists on sympathetic and parasympathetic nerve activity. J Cardiovasc Pharmacol 1990; 15: S75-S85

[16] Verberne AJM, Rand MJ. Effect of urapidil on $\beta$-adrenoceptors of rat atria". Eur J Pharmacol 1985; 108: 193-196.

[17] Dooley M, Goa KL. Drugs. Urapidil. A reappraisal of its use in the management of hypertension.1998;56:929-955

[18] Wacker J, Werner P, Walter-Sack I, Bastert G. Treatment of hypertension in patients with pre-eclampsia: a prospective parallel-group study comparing dihydralazine with urapidil. Nephrol Dial Transplant. 1998;13,318-325

[19] Ebrantil.spc.http://db.cbg-meb.nl/Pars/h101891.pdf

[20] Hall et al. Sedative, analgesic and cognitive effects of clonidine infusions in humans. British Journal of Anaesthesia 2001;86:5-11

[21] Catapres.spc.http://www.medicines.org.uk/emc/medicine/283

[22] Murphy MB, Murray C, Shorten GD. Fenoldopam-a selective peripheral dopaminereceptor agonist for the treatment of severe hypertension. N Engl J Med. 2001; 345:1548-57

[23] Fenves AZ, Ram CV. Drug treatment of hypertensive urgencies and emergencies. Semin Nephrol. 25: 2005; 272-280

[24] DiPette DJ et al. Enalaprilat, an intravenous angiotensinconvertingenzyme inhibitor, inhypertensive crises Clin Pharmacol Therap 1985; 2: 199-204

[25] Dilceren.spc. http://www.medicines.org.uk/emc/medicine/5581

[26] Clevidipine.spc.http://www.medicines.org.uk/emc/medicine/5581

[27] Phung OJ, Baker WL, White CM et al. Clevidipine: An intravenous dihydropyridine calcium-channel blocker for the treatment of acute hypertension. Formulary 2009;44:102-107

[28] Cleviprex.spc.http://www.medicines.org.uk/emc/medicine/28128

[29] Isoptin.spc.http://www.medicines.ie/medicine/11962/SPC/

[30] Esmocard.spc.http://db.cbg-meb.nl/mri/spc/nlh-0779-002. pdf

[31] Betaloc.spc.http://www.medicines.org.uk/emc/medicine/152

[32] Trandate.spc.https://www.medicines.org.uk/emc/medicine/4297

[33] Regitin.http://www.old.health.gov.il/units/pharmacy/trufot/ alonim/4502.pdf

[34] Varon J. Diagnosis and management of labile blood pressure during acute cerebrovascular accidents and other hypertensive crises. Am J Emerg Med 2007; 25: 949-995 
[35] European Stroke Initiative: European Stroke Initiative recommendations for stroke management. European Stroke Council, European Neurological Society and European Federation of Neurological Societies. Cerebrovasc Dis 2000;10:335-351

[36] The European Stroke Initiative Executive Committee and the EUSI Writing Committee: European Stroke Initiative Recommendations for Stroke Management - Update 2003. Cerebrovascular Disease 2003;16:311-337

[37] Steiner T, Kaste M, Forsting M, Mendelow D, Kwiecinski H, Szikora I, Juvela S, Marchel A, Chapot R, Cognard C, Unterberg A, Hacke W: Recommendations for the management of intracranial haemorrhage-part I: spontaneous intracerebral haemorrhage. The European Stroke Initiative Writing Committee and the Writing Committee for the EUSI Executive Committee. Cerebrovasc Disease 2006;22:294-316

[38] Devlin JW, Seta ML, Kanji S, Somerville AL. Fenoldopam versus nitroprusside for the treatment of hypertensive emergency. Ann Pharmacother. 38: 2004; 755-759 
